\title{
Generation of Non-Gaussian Wide-Sense Stationary Random Processes with Desired PSDs and PDFs
}

\author{
Milad Johnny \\ Electrical and Communication Engineering, Tehran, Iran. \\ Email: M_Johnny@elec.iust.ac.ir \\ Received April 17 $7^{\text {th }}, 2012$; revised May 23 ${ }^{\text {rd }}, 2012$; accepted July $2^{\text {nd }}, 2012$
}

\begin{abstract}
This paper describes a new method to generate discrete signals with arbitrary power spectral density (PSD) and first order probability density function (PDF) without any limitation on PDFs and PSDs. The first approximation has been achieved by using a nonlinear transform function. At the second stage the desired PDF was approximated by a number of symmetric PDFs with defined variance. Each one provides a part of energy from total signal with different ratios of remained desired PSD. These symmetric PDFs defined by sinusoidal components with random amplitude, frequency and phase variables. Both analytic results and examples areincluded. The proposed scheme has been proved to be useful in simulations involving non-Gaussian processes with specific PSDs and PDFs.
\end{abstract}

Keywords: Asymmetrical PDF; Signal Representation; Ergodicity; Gaussian Variables; Non-Infinity Devisable; Stealth Objects

\section{Introduction}

Generating and representing of a Gaussian variable with desired PSD is an easy job. A usual method is to use simple linear time invariant filter with a Gaussian random variable as an input. The problem of generating correlated non-Gaussian random series has been of great interest during the last three decades in connection with the simulation of processes in communication systems such as radar, sonar and speech. Furthermore, it can be applicable in recent military approaches. Groups of stealth aircrafts generate random noise with $K$ distribution to be hidden under clutter noise from active radars. The generation of non-Gaussian signals has given rise to a renewed interest in the mechanical vibration fields and defense industries for some reasons. The realization of many surface transportation and wave environments are non-Gaussian, also the development of shaker control systems that can replicate long time histories are non-Gaussian. Many original current shaker control systems, for generating random vibration tests generate only Gaussian random noise. However, waveform replication techniques now allow the reproduction of any waveforms whose characteristics are within the bounds of a shaker $[1,2]$. Most of the approaches suggested involve the non-linear transformation of colored Gaussian random series or use linear transformation of non-Gaussian no correlated time series. All these methods require a complicated optimization procedure to achieve the de- sired properties $[3,4]$. The zero memory non-linear ZMNL in [3] is chosen so that the desired distribution is exactly realized and the digital filter is designed so that the specific auto-covariance is closely approximated. In this method, the autocorrelation of sequence at the output of the filter may be negative hence the method can fail because in this case we should calculate several hundreds or even thousands of unknowns, on the other hand ergodicity has not been mentioned in this paper. In addition, the method is too complex to be implemented but it can be helpful in this paper for first approximation with some promotion. In [5], a correlated Gaussian random process is multiplied by a modulating sequence. The main problem is in determining the modulating sequence, which can be difficult if not impossible. Furthermore, the generated sequence is not ergodic. In [6], a nonlinearity is used and due to the nonlinearities in these schemes, it seems that the results cannot be extended to the general cases such as multidimensional and multichannel random processes. The method suggested in [7], is based on [3] and decreases some problems and produces wide-sense stationary and ergodic sequences asymptotically or as the number of generated data goes to infinity. In [8] proposed a method that decouples the problem into two separate ones for some signal characteristics such as even and infinitely divisible first-order PDF, but it can generate every continuous PSDs. The method can be limited because the Hankel transform of every PDFs cannot be 
calculated easily. The restriction of an even PDF limits us to generate vast major PDFs such as Rayleigh, Nakagami, flicker and square Gaussian noises. The assumption of an infinitely divisible PDF may be restrictive too. However, the strategy mentioned in [8] is a constructive tool to be used under some modifications. In this paper, we divide desired PDF with a number of symmetric and infinitely divisible ones that these PDFs depend on the first estimation. In contradiction to [8] there is not any restriction on the PDF. This paper consists of five section, in section 2 a basic function which has been derived from [8] is considered and extended to more general form and the nonlinear transform function is described for closed form first approximation. In section 3, with some assumption we prove that this function has ergodicity properties in both means and variances and the way to compute a nonlinear transform function has been described. In section 4, a complex signal with asymmetric PDF and desired PSD are generated. The conclusion has been given in section 5 .

\section{Definition and Properties}

We propose a new method to generate arbitrary continuous PSDs and first-order PDFs. In this method, one can generate a discrete signal by dividing the desired PDF into a number of symmetric PDFs. In general form we define $Z[n]$ for $-\infty<n<\infty$ as follows

$$
\begin{aligned}
Z[n]= & \sum_{i=1}^{M}\left\{A_{i} \cos \left(2 \pi F_{i} n+\varphi_{i}\right)\right. \\
& \left.+j B_{i} \cos \left(2 \pi F_{i}^{\prime} n+\varphi_{i}^{\prime}\right)+\frac{L_{n}}{M}+j \frac{L_{n}^{\prime}}{M}\right\} \\
S=\operatorname{Re} & \{S\}+j \operatorname{Im}\{S\}=x+j y
\end{aligned}
$$

where $\operatorname{Re}\{\}$ and show real and imaginary parts of $S$ respectively that are independent from each other. In (1) $A_{i}, B_{i}, \varphi_{i}, \varphi_{i}^{\prime}, F_{i}, F_{i}^{\prime}, L_{n}$ and $L_{n}^{\prime}$ are random variables with the following definitions

$$
\begin{aligned}
L_{n} & \sim p_{X}(x) \& L_{n}^{\prime} \sim p_{Y}(y) \\
\varphi_{i} & \sim U(-\pi, \pi), \varphi_{i}^{\prime} \sim U(-\pi, \pi) \\
F_{i} & \sim p_{F}(f) \& F_{i}^{\prime} \sim p_{F^{\prime}}(f) 0 \leq F \leq \frac{1}{2} \\
A & \sim p_{A}(a), B \sim p_{B}(b)
\end{aligned}
$$

In all sections of this article, we assume $x, L$ and $y, L^{\prime}$ each have equal distributions. By considering the above-mentioned relations the following are shown in appendix

1) The mean of $Z[n]$ is

$$
E[Z[n]]=E[x]+j E[y]
$$

The mean of every PDFs is a deterministic value and it forces the PSD to have a $\delta$ function in zero. Therefore, it can be subtracted from $Z[n](E[x]=0, E[y]=0)$.

1) The random process is the wide sense stationary with the following autocorrelation function

$$
\begin{aligned}
& R_{Z}(n, n+r)=\frac{M}{2} E\left[A^{2}\right] \int_{0}^{\frac{1}{2}} \cos (2 \pi f r) p(f) \mathrm{d} f \\
& +\frac{M}{2} E\left[B^{2}\right] \int_{0}^{\frac{1}{2}} \cos \left(2 \pi f^{\prime} r\right) p\left(f^{\prime}\right) \mathrm{d} f^{\prime}+\mathfrak{B}_{r}+\mathfrak{B}_{r}^{\prime}
\end{aligned}
$$

where $\mathfrak{B}_{r}, \mathfrak{B}_{r}^{\prime}$ are $E\left[L_{n} L_{n+r}\right], E\left[L_{n}^{\prime} L_{n+r}^{\prime}\right]$ respectively. Actually, the power spectral density is equal to

$$
\begin{aligned}
P_{Z}(f) & =\frac{M}{4} E\left[A^{2}\right] p_{F}(f)+\frac{M}{4} E\left[B^{2}\right] p_{F^{\prime}}(f) \\
& +\sum_{r=-\infty}^{\infty}\left[\mathfrak{B}_{r}+\mathfrak{B}_{r}^{\prime}\right] \mathrm{e}^{-j 2 \pi f r} 0 \leq f \leq \frac{1}{2}
\end{aligned}
$$

Now we can use the nonlinear transform function in order to achieve the first approximation of the desired spectrum and the first order statistical probability density function. This spectrum can be converged exactly on desired one by using $\frac{M}{4} E\left[A^{2}\right] p_{F}(f)$ and $\frac{M}{4} E\left[B^{2}\right] p_{F^{\prime}}(f)$ terms, with negligible deviation from main PDF. Because of positive definition of $p_{F}(f)$ and $p_{F^{\prime}}(f)$ we should have

$$
P_{S}(f)-\sum_{r=-\infty}^{\infty}\left[\mathfrak{B}_{r}+\mathfrak{B}_{r}^{\prime}\right] \mathrm{e}^{-j 2 \pi f r} \geq 0,0 \leq f \leq \frac{1}{2}
$$

where $P_{S}(f)$ is the desired power spectrum.

3) The PDF of $A_{i}$ and $B_{i}$ can be written as

$$
\begin{aligned}
& p_{a}(A)=a \int_{0}^{\infty}\left[\left.\Psi_{\operatorname{Re}\{Z[n]\}}\right|_{L, L^{\prime}}\right]^{\frac{1}{M}} J_{0}(\omega a) \omega \mathrm{d} \omega \\
& p_{b}(B)=b \int_{0}^{\infty}\left[\left.\Psi_{\operatorname{Im}\{Z[n]\}}\right|_{L, L^{\prime}}\right]^{\frac{1}{M}} J_{0}(\omega b) \omega \mathrm{d} \omega
\end{aligned}
$$

where $\left.\Psi_{\operatorname{Re}\{Z[n]\}}\right|_{L, L^{\prime}}$ and $\left.\Psi_{\operatorname{Im}\{Z[n]\}}\right|_{L, L^{\prime}}$ are the two

characteristic functions for symmetric PDFs with proper variance and zero mean, for a Gaussian $N\left(0, \sigma^{2}\right)$ we have

$$
\left.\Psi_{\operatorname{Re}\{Z[n]\}}\right|_{L, L^{\prime}}=\left.\Psi_{\operatorname{Im}\{Z[n]\}}\right|_{L, L^{\prime}}=\mathrm{e}^{\frac{-\sigma^{2} \omega^{2}}{2}}
$$

therefore

$$
p_{a}(A)=a \int_{0}^{\infty} \exp \left(-\frac{\sigma^{2} \omega^{2}}{2 M}\right) J_{0}(\omega a) \omega \mathrm{d} \omega
$$

and we can solve the above relation by using Hankel transformation of $\exp \left(-\frac{\sigma^{2} \omega^{2}}{2 M}\right)$, hence 


$$
p_{a}(A)=\frac{a}{\sigma^{2} / M} \exp \left[-\frac{a^{2} / 2}{\sigma^{2} / M}\right]
$$

similarly, we have

$$
p_{B}(b)=\frac{b}{\sigma^{2} / M} \exp \left[-\frac{b^{2} / 2}{\sigma^{2} / M}\right]
$$

In order to use the nonlinear transform function, consider the following relation between two random variables of $x$ and $t$

$$
L \sim p_{X}(x)=p_{T}(t)\left|\frac{\mathrm{d} t}{\mathrm{~d} x}\right|
$$

where $p_{X}(x)$ and $p_{T}(t)$ are probability density functions for $x$ and $t$ random variables respectively. Similarly, for both random variables of $y$ and $t^{\prime}$ we have

$$
L^{\prime} \sim p_{Y}(y)=p_{T^{\prime}}(t)\left|\frac{\mathrm{d} t^{\prime}}{\mathrm{d} y}\right|
$$

It is understood that in Equations (16) and (17), $t, t^{\prime}$ stands for $t=g_{1}^{-1}(x)$ and $t^{\prime}=g_{2}^{-1}(y)$, the $x=g_{1}(t)$ and $y=g_{2}\left(t^{\prime}\right)$ are the density functions which are dependent on $x$ and $y$ variables. In this paper all the relations and calculations on $L, t$ variables can be modified to $L^{\prime}, t^{\prime}$ variables.

If $L=g_{1}(t)$ is not monotonically increasing, the function must be broken into parts, and each part is handled separately. For the purposes of this article $x=g_{1}(t)$ will be restricted to its derivation function. Therefore, the sign of $\mathrm{d} x / \mathrm{d} t$ can be negative or positive. The conversion of a realization of $t$ to $x$ using a nonlinear function will always produce a PSD for $x$ that is different from the PSD of $t$. The effect is to add harmonics to all the Fourier components of $t$. This will make the transformed data appear "whiter" than the original data $[9,10]$. However, most of the spectral information is contained in the zero crossings which are preserved, and if the nonlinear- ity is not too great, the spectral change is usually accept- able and the PSD of $X$ will be near the PSD of $t$. The practical utility of these results is that the PSD may be approximated from (9) by using nonlinear transform function on $L_{n}, L_{n}^{\prime}$ and is converged to exact form by choosing the PDF of frequencies $p_{F}(f)$ and $p_{F^{\prime}}\left(f^{\prime}\right)$ properly.

\section{Ergodicity Properties, Nonlinear Function Synthesizing}

The main problem in applications of stochastic processes is the estimation of various statistical parameters in terms of real and imaginary parts. It is clear that the basic Equation (1) contains two mathematical terms, the first one is

$$
\sum_{i=1}^{M}\left\{A_{i} \cos \left(2 \pi F_{i} n+\varphi_{i}\right)+j B_{i} \cos \left(2 \pi F_{i}^{\prime} n+\varphi_{i}^{\prime}\right)\right\}
$$

which its ergodicity of mean and variance has been proven in [1]. The second term is $L_{n}+j L_{n}^{\prime}$, where $L_{n} \sim p_{X}(x)$, $L^{\prime} \sim p_{Y}(y)$ and $L_{n}=g_{1}(t), L_{n}^{\prime}=g_{2}\left(t^{\prime}\right)$ therefore if $t, t^{\prime}$ has an ergodic properties and $g_{1}(t)<\infty$,

$g_{2}\left(t^{\prime}\right)<\infty$ both $L_{n}$ and $L_{n}^{\prime}$ have ergodicity properties too. In other words, Equation1 under certain conditions has ergodicity properties. Now it is clear, that

the main problem is to synthesize $g_{1}(t)$ and $g_{2}\left(t^{\prime}\right)$ under the limitation of Equation (10). The total power spectrum can be divided as follows

$$
P_{S}(f)=P_{S_{1}}(f)+P_{S_{2}}(f)
$$

where $P_{S_{1}}(f)$ and $P_{S_{2}}(f)$ are the power spectrum of real and imaginary parts respectively. Therefore, we can assume

$$
\left\{\begin{array}{l}
P_{S_{1}}(f)-\sum_{r=-\infty}^{\infty} \mathfrak{B}_{r} \mathrm{e}^{-j 2 \pi f r} \geq 0 \\
P_{S_{2}}(f)-\sum_{r=-\infty}^{\infty} \mathfrak{B}_{r}^{\prime} \mathrm{e}^{-j 2 \pi f r} \geq 0
\end{array}\right.
$$

Both strategies on imaginary and real parts are like each other. Equation (18) can be simplified as

$$
P_{S_{1}}(f)-\left\{\mathfrak{B}_{0}+2 \sum_{r=0}^{\infty} \mathfrak{B}_{r} \cos (2 \pi f r)\right\} \geq 0
$$

and

$$
\begin{aligned}
& \mathfrak{B}_{r}=E\left[L_{n} L_{n+r}\right] \leq 2 \int_{0}^{\frac{1}{2}} P_{S_{1}}(f) \cos (2 \pi f r) \mathrm{d} f \\
& \mathfrak{B}_{0}=E\left[L_{n}^{2}\right] \leq 4 \int_{0}^{\frac{1}{2}} P_{S_{1}}(f) \mathrm{d} f
\end{aligned}
$$

If $g_{1}(t) \leq\{t-\mu\}$ and $t$ has a Gaussian distribution

$$
\mathfrak{B}_{r}=E\left[L_{n} L_{n+r}\right]<E\left[t_{n} t_{n+r}\right]
$$

Now we propose a computational method to generate a proper function of $g_{1}(t)$, set $g_{1}(t=\mu)=\operatorname{mean}(x)$ where we select $\mu$ as large as possible, considering small positive value for $\Delta t$, we have

for $t<\mu$

while $-|\gamma|<t \leq|\gamma|$

$\left|\frac{\mathrm{d} x}{\mathrm{~d} t}\right|=p_{X}\left(x=g_{1}(t)\right)\left[\frac{1}{\sqrt{2 \pi \sigma^{2}}} \exp \left(-\frac{(t-\mu)^{2}}{2 \sigma^{2}}\right)\right]^{-1}$

$g_{1}(t-\Delta t)=g_{1}(t)-\Delta t|\mathrm{~d} x / \mathrm{d} t|$

if $g_{1}(t-\Delta t) \geq\{t-\mu-\Delta t \operatorname{sign}(t)\}$

$g_{1}(t-\Delta t)=g_{1}(t)+\Delta t|\mathrm{~d} x / \mathrm{d} t|$

end

$$
t=t-\Delta t
$$


end

end

Where $\gamma$ is a large number which $\gamma \gg \mu$, Figure 1 shows nonlinear function synthesizing to generate uniform PDF in $[-0.5,0.5]$ interval. It is clear that generated function guarantees the Equations (20) and (21).

\section{Some Examples of Generating Asymmetric and PDF with Desired PSD}

\subsection{One Dimensional Real Signal}

Consider the representation of a WSS Rayleigh random process with the variance of $\left(2-\frac{\pi}{2}\right) \sigma^{2}$, mean given by $\sigma \sqrt{\pi / 2}$, and a given continuous PSD. To represent and generate this process we need determining a nonlinear transform function to convert Gaussian distribution of $t$ to Rayleigh PDF, the PDFs of the amplitude and frequency can be calculated after the first approximation of desired PSD. Figure 2 shows a nonlinear transform function to convert Gaussian with shaped spectrum to Rayleigh one. Figure 3 depicts the Rayleigh PDF, the blue curve in Figure 4 shows the first approximated power spectral density and the red dashed line shows desired spectrum. We define the following error function

$$
\epsilon_{1}=\int_{0}^{1 / 2}\left\{P_{S}(f)-\sum_{r=-\infty}^{\infty} \mathfrak{B}_{r} \mathrm{e}^{-j 2 \pi f r}\right\} \mathrm{d} f
$$

This error function can be decreased by the use of sinusoidal terms

$$
\sum_{i=1}^{M}\left\{A_{i} \cos \left(2 \pi F_{i} n+\varphi_{i}\right)\right\}
$$

where

$$
\frac{M}{4} E\left[A^{2}\right] p_{F}(f)=P_{S}(f)-\sum_{r=-\infty}^{\infty} \mathfrak{B}_{r} \mathrm{e}^{-j 2 \pi f r}
$$

and

$$
\int_{0}^{1 / 2} \frac{M}{4} E\left[A^{2}\right] p_{F}(f) \mathrm{d} f=\frac{M}{4} E\left[A^{2}\right]=\epsilon_{1}
$$

Therefore, the frequency PDF can be calculated as the following relation

$$
p_{F}(f)=\frac{P_{S}(f)-\sum_{r=-\infty}^{\infty} \mathfrak{B}_{r} \mathrm{e}^{-j 2 \pi f r}}{\epsilon_{1}}
$$

also

$$
p_{a}(A)=\frac{a}{\sigma^{2} / M} \exp \left[-\frac{1 / 2 a^{2}}{\sigma^{2} / M}\right]
$$

The average of 10,000 realizations of a periodogram along with the true PSD (indicated by the black curve) is shown in Figure 4. It is interesting to note that the generated PDF has less accuracy than what is calculated before adding sinusoidal term and it is depicted in Figure 5. First approximation play an important role in having a more accurate results.

\subsection{Two Dimensional Complex Signal}

Consider the WSS complex signal $S=x+j y$ with ar-

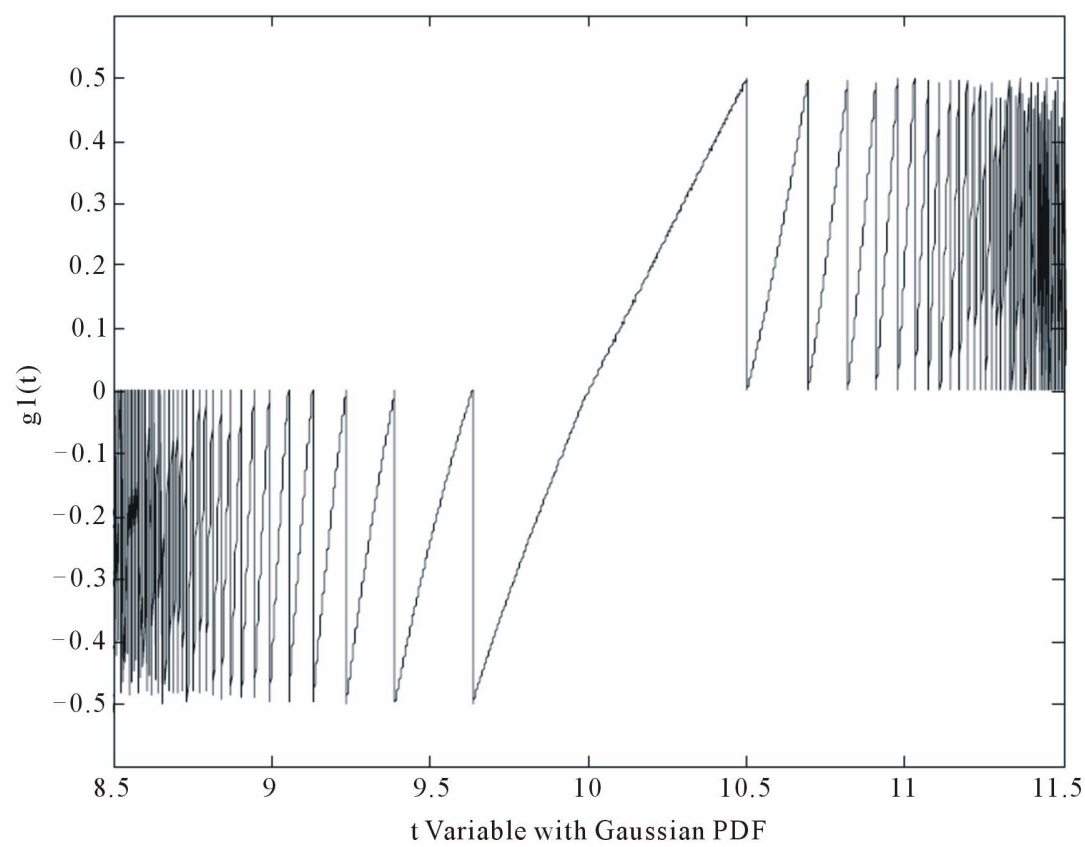

Figure 1. Proper nonlinear transform function $g_{1}(t), t \sim N\left(10, \sigma^{2}=0.25\right)$ and $x=g_{1}(t) \sim U(-0.5,0.5)$. 


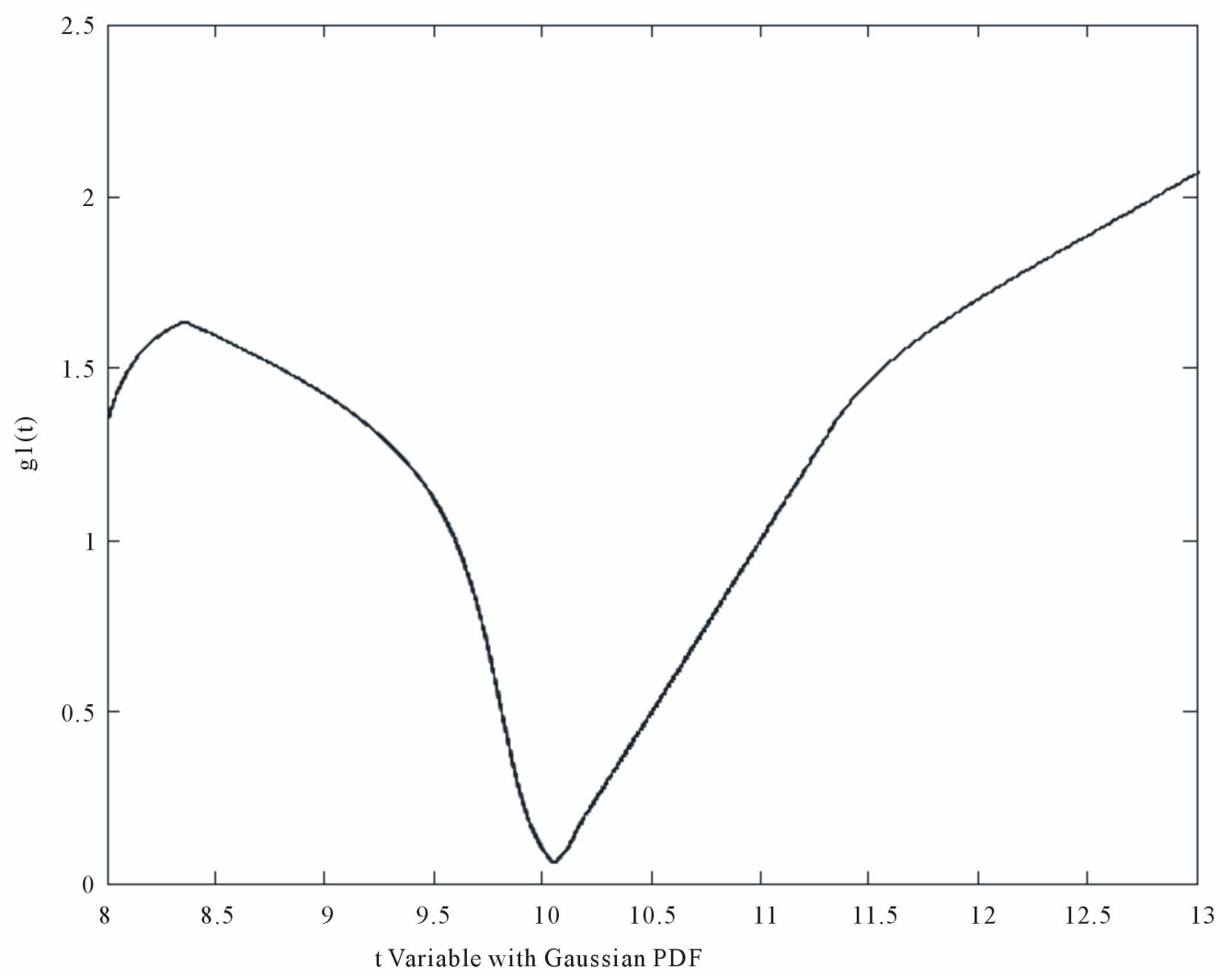

Figure 2. Nonlinear transform function converting non zero mean Gaussian PDF to Rayleigh random process.

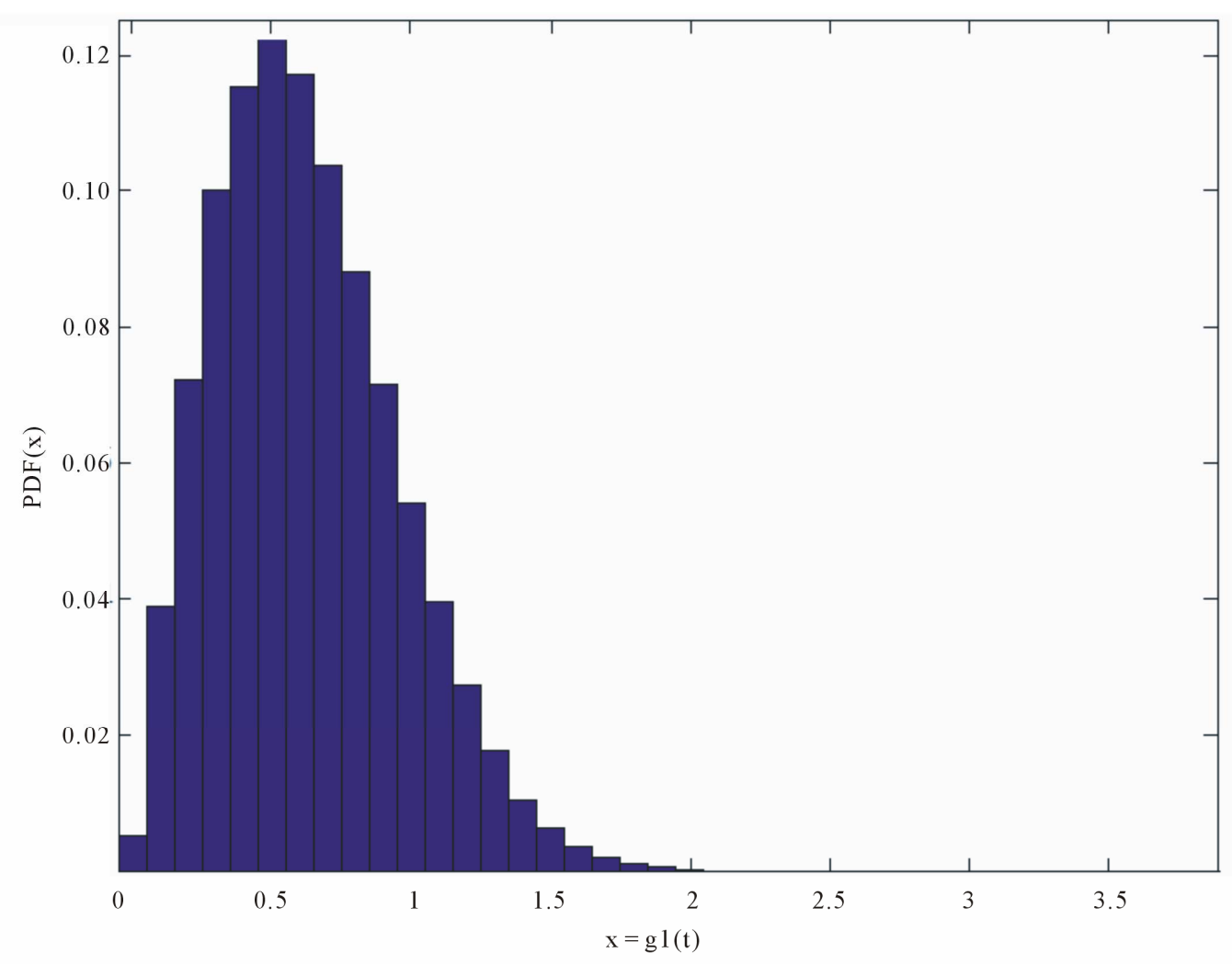

Figure 3. Generated Rayleigh random process after applying $g(t)$ on Gaussian PDF. 


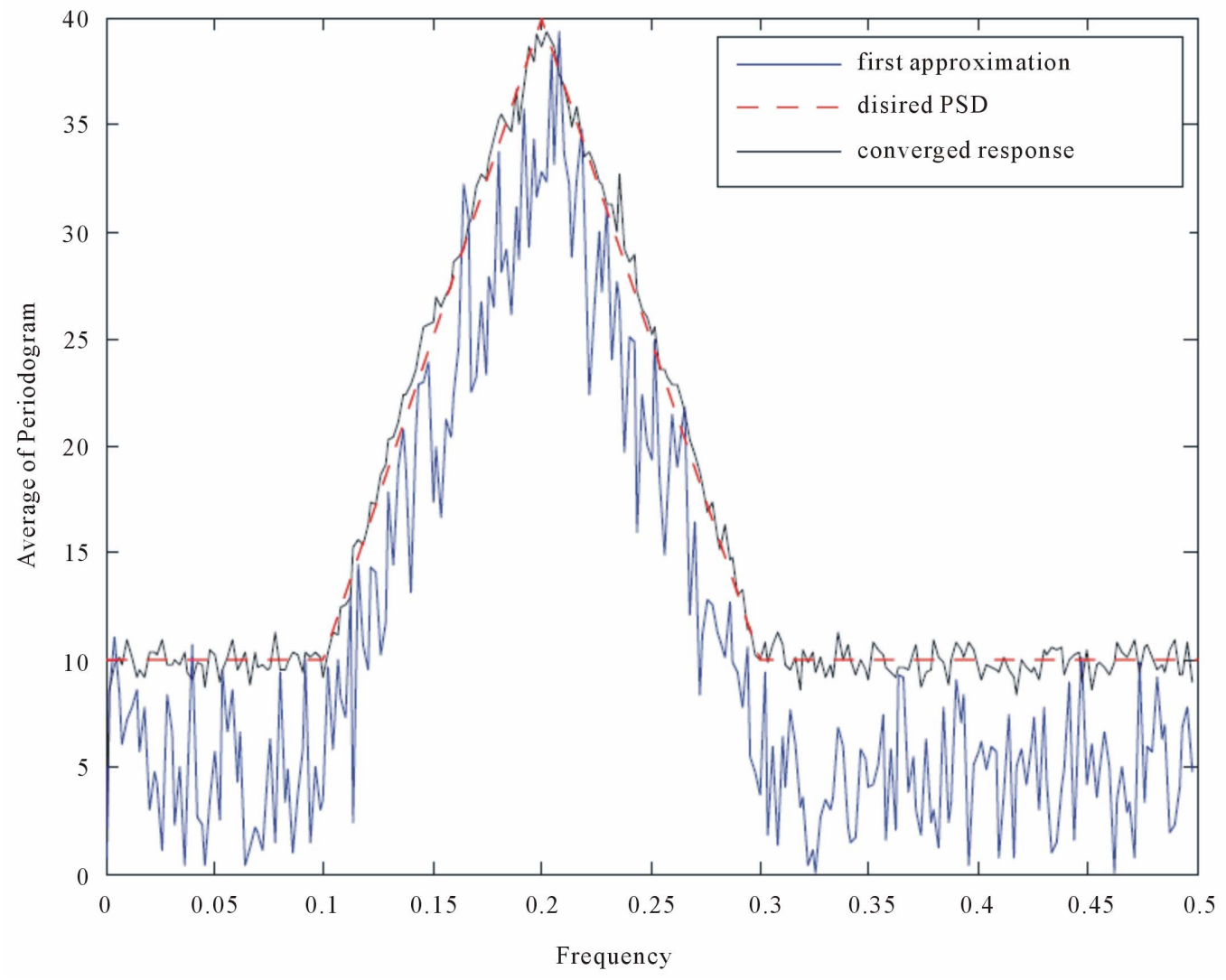

Figure 4. Power spectral density synthesizing.

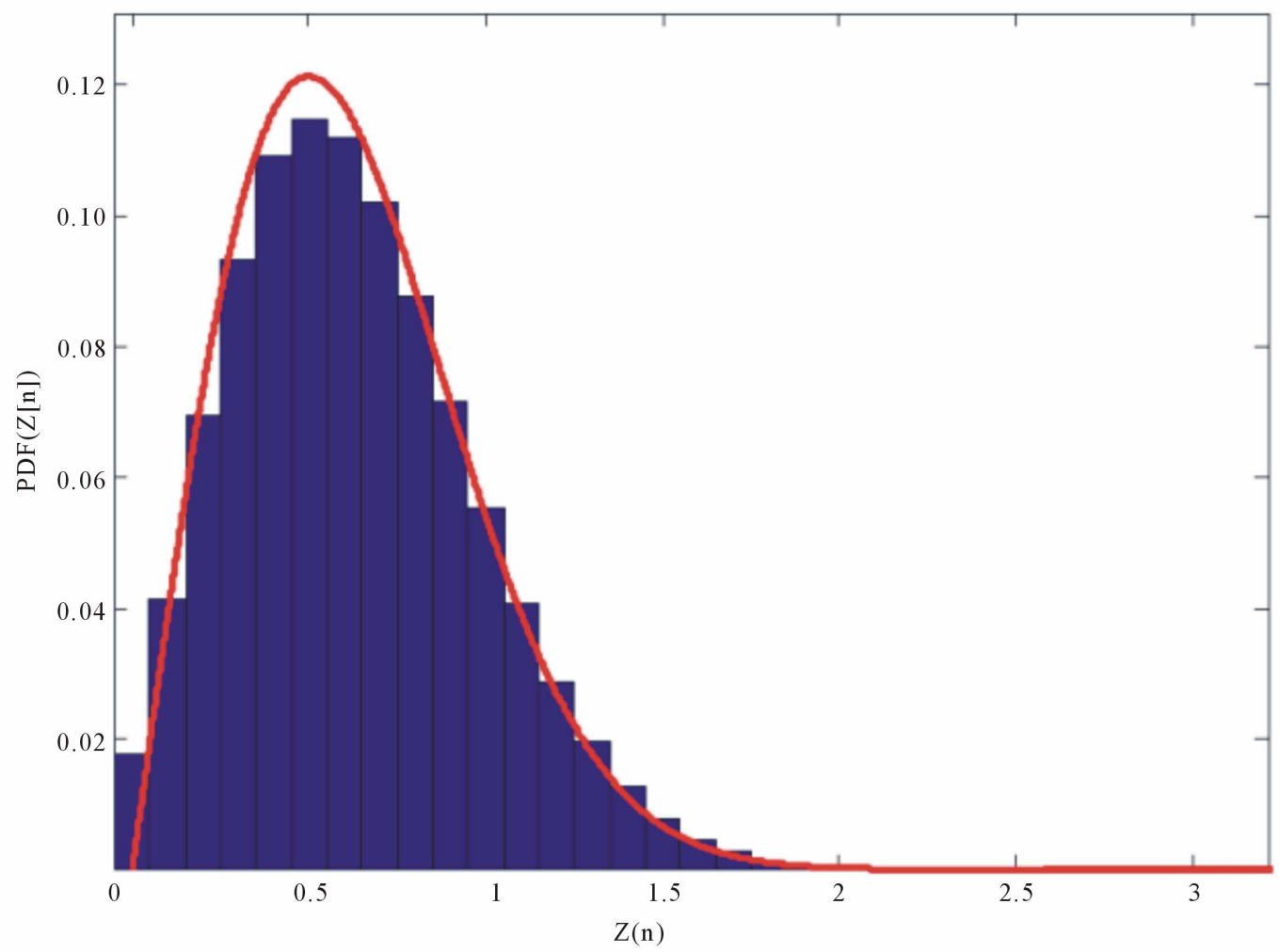

Figure 5. First order probability density function of $Z[n]$, red line depicts Rayleigh PDF. 
bitrary power spectral density, where $x$ and $y$ are independent from each other and represent the real and complex parts of $S$ respectively. Both $x$ and $y$ have desired PDF and every one generates a part of total power spectrum which depends on its variance. Therefore $p_{S}(s)=p_{X}(x) p_{Y}(y)$, where $p_{S}(s)$ is the PDF of a complex signal and $p_{X}(x), p_{Y}(y)$ represent the PDF of real and imaginary parts of the signal. Figure 6 shows a joint PDF of $x$ and $y$. If we consider

$$
\begin{aligned}
& \mathfrak{B}_{r}=E\left[L_{n} L_{n+r}\right] \leq 2 \int_{0}^{\frac{1}{2}} P_{S_{1}}(f) \cos (2 \pi f r) \mathrm{d} f \\
& \mathfrak{B}_{0}=E\left[L_{n}^{2}\right] \leq 4 \int_{0}^{\frac{1}{2}} P_{S_{1}}(f) \mathrm{d} f
\end{aligned}
$$

and

$$
\begin{aligned}
& \mathfrak{B}_{r}^{\prime}=E\left[L_{n}^{\prime} L_{n+r}^{\prime}\right] \leq 2 \int_{0}^{\frac{1}{2}} P_{S_{2}}(f) \cos (2 \pi f r) \mathrm{d} f \\
& \mathfrak{B}_{0}^{\prime}=E\left[L_{n}^{\prime 2}\right] \leq 4 \int_{0}^{\frac{1}{2}} P_{S_{2}}(f) \mathrm{d} f
\end{aligned}
$$

where

$$
\begin{aligned}
& P_{S_{1}}(f)=\frac{\operatorname{var}(x) P_{S}(f)}{\operatorname{var}(x)+\operatorname{var}(y)} \\
& \& P_{S_{2}}(f)=\frac{\operatorname{var}(y) P_{S}(f)}{\operatorname{var}(x)+\operatorname{var}(y)}
\end{aligned}
$$

After applying the algorithm that was mentioned in
Section 3 we can achieve to proper nonlinear transform function. Figure 7 shows generated PDF and Figure 8 depicts imaginary and real parts of this discrete signal. The power spectrum can be converged to its correct response by considering proper PDF for both $F$ and $F^{\prime}$ values as follows

$$
\begin{aligned}
& \int_{0}^{1 / 2} \frac{M}{4} E\left[A^{2}\right] p_{F}(f) \mathrm{d} f=\frac{M}{4} E\left[A^{2}\right]=\epsilon_{1} \\
& \int_{0}^{1 / 2} \frac{M}{4} E\left[B^{2}\right] p_{F^{\prime}}\left(f^{\prime}\right) \mathrm{d} f^{\prime}=\frac{M}{4} E\left[B^{2}\right]=\epsilon_{2}
\end{aligned}
$$

Therefore

$$
\begin{aligned}
& p_{F}(f)=\frac{P_{S_{1}}(f)-\sum_{r=-\infty}^{\infty} \mathfrak{B}_{r} \mathrm{e}^{-j 2 \pi f r}}{\epsilon_{1}} \\
& p_{F^{\prime}}\left(f^{\prime}\right)=\frac{P_{S_{2}}(f)-\sum_{r=-\infty}^{\infty} \mathfrak{B}_{r}^{\prime} \mathrm{e}^{-j 2 \pi f r}}{\epsilon_{2}}
\end{aligned}
$$

Generated and desired power spectral density also has been shown in Figure 9.

\section{Conclusion}

A new method has been presented which can generate a complex WSS random process with a desired PSD and a given first-order PDF. One of the advantages of this method is that we have no limitation on PDF to be infi-

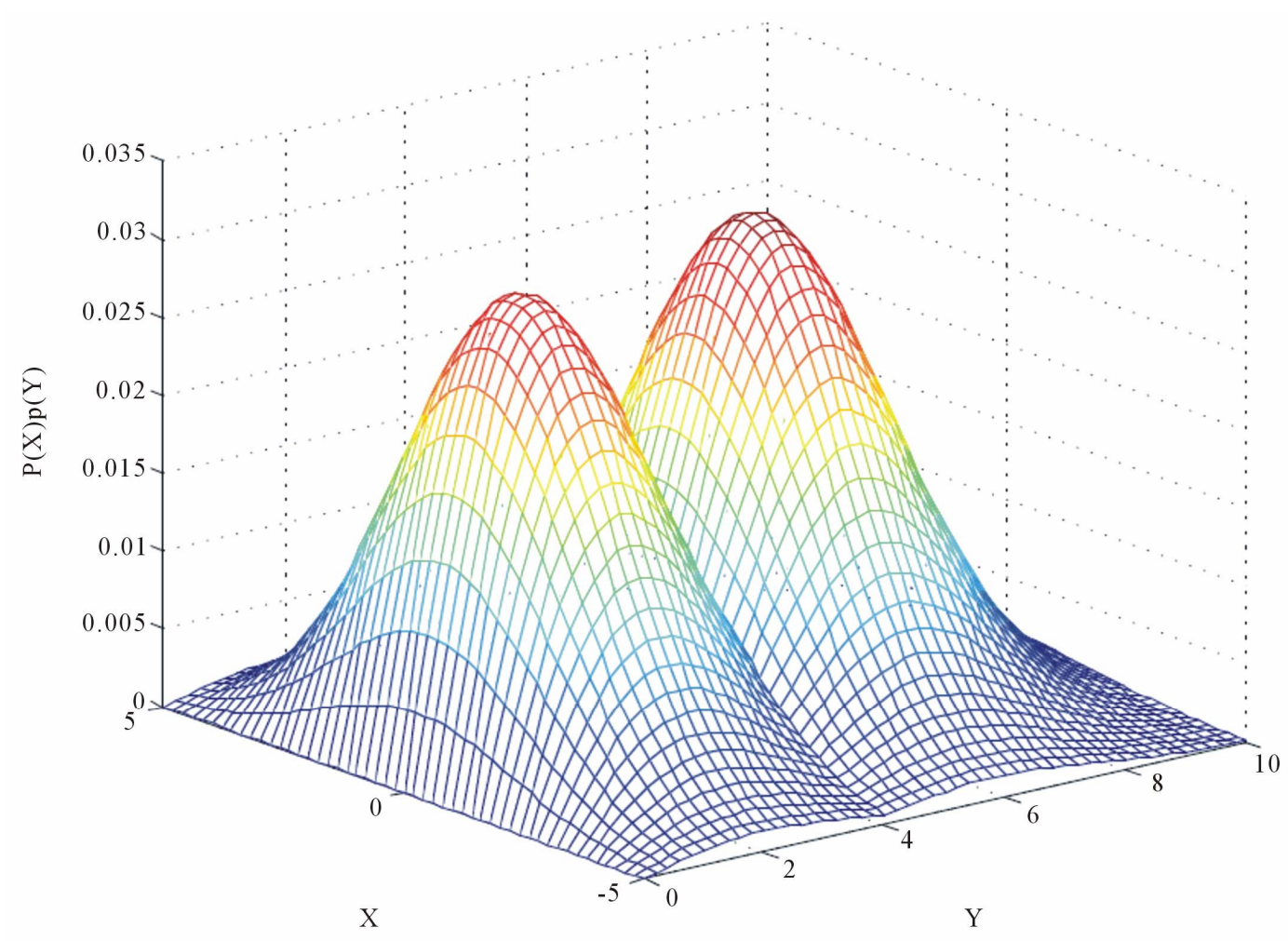

Figure 6. Joint PDF of $x$ and $y$. 


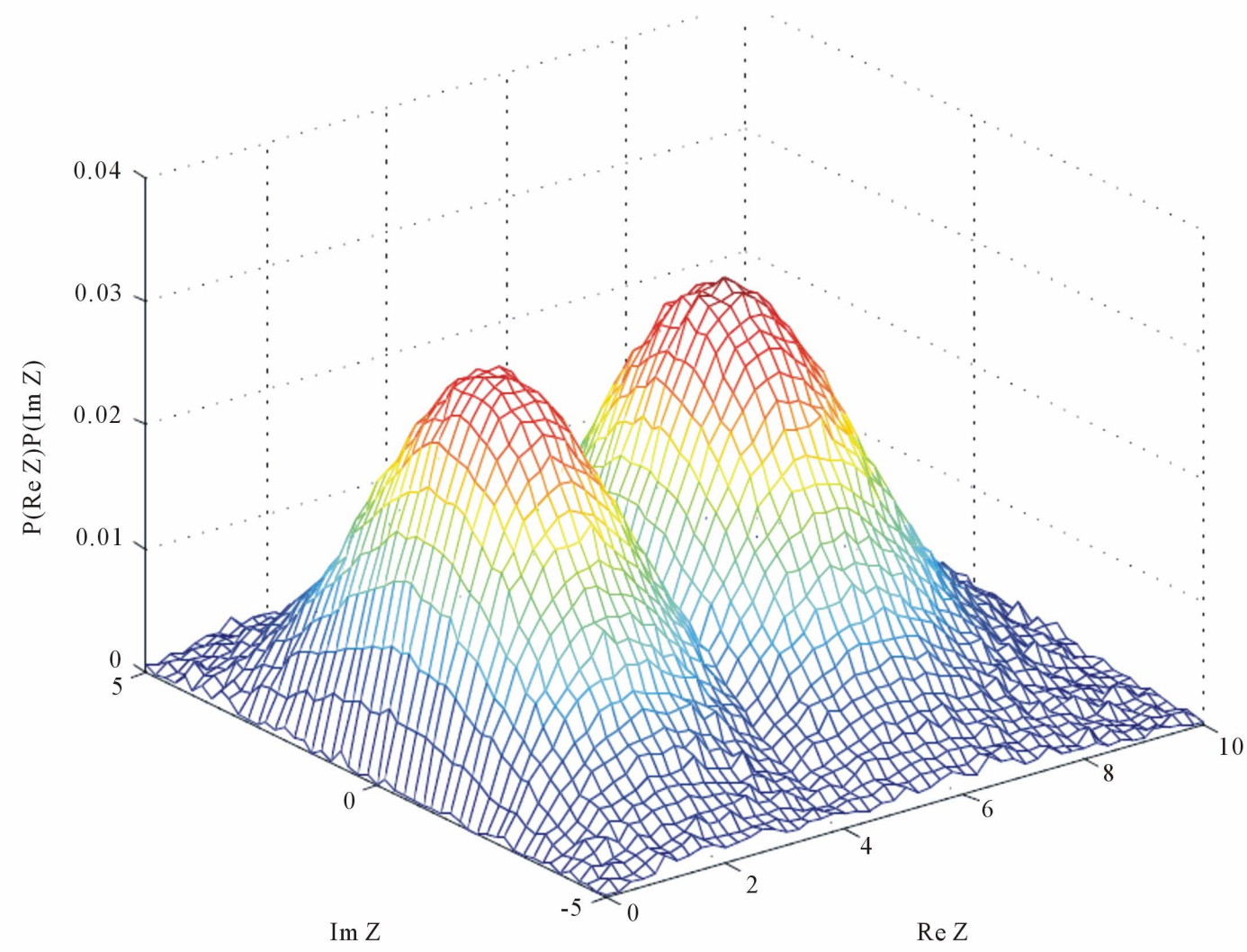

Figure 7. Generated joint $\operatorname{PDF}$ of $\operatorname{Re}\{Z\}$ and $\operatorname{Im}\{Z\}$.
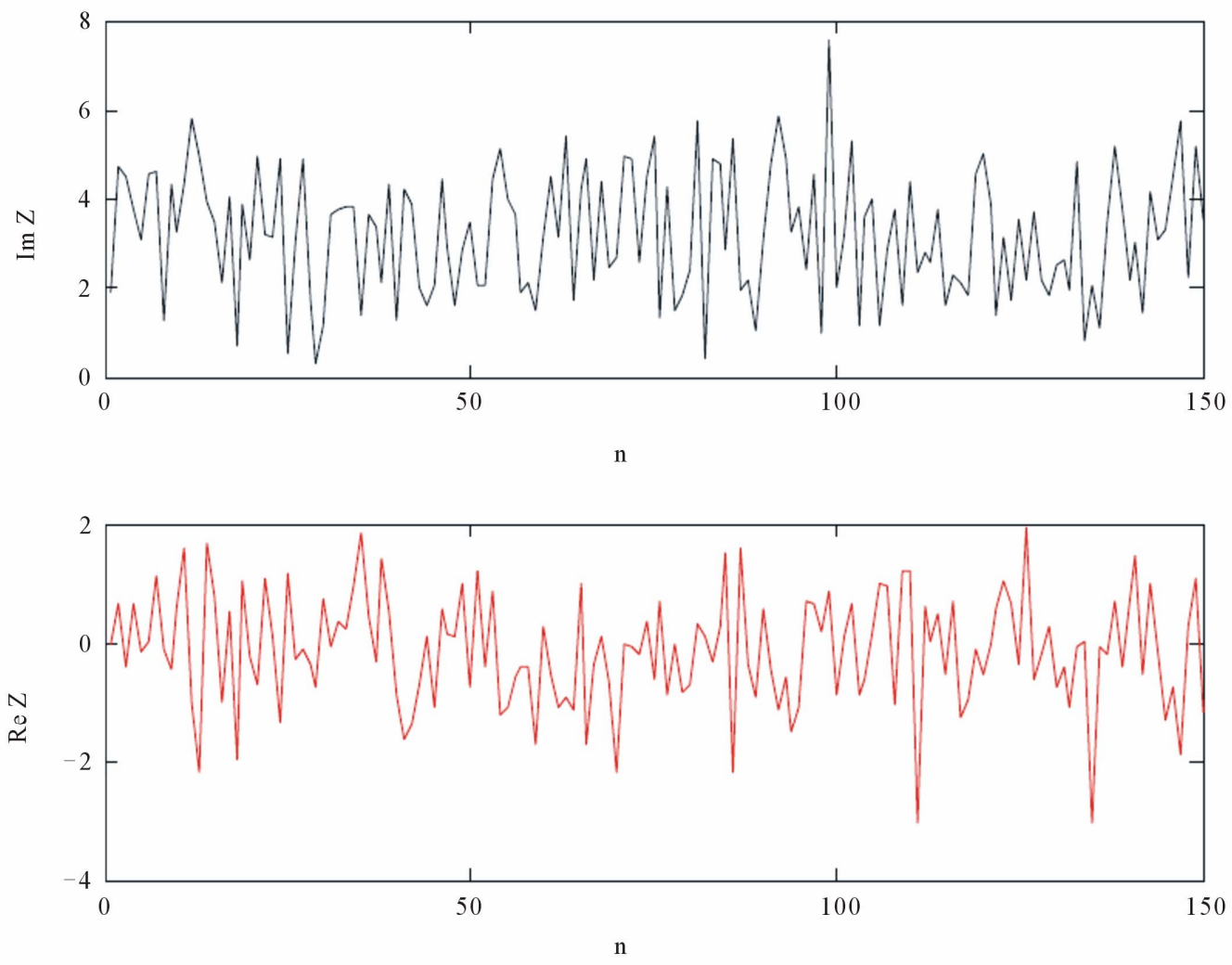

Figure 8. Discrete imaginary and real parts of generated signal. 


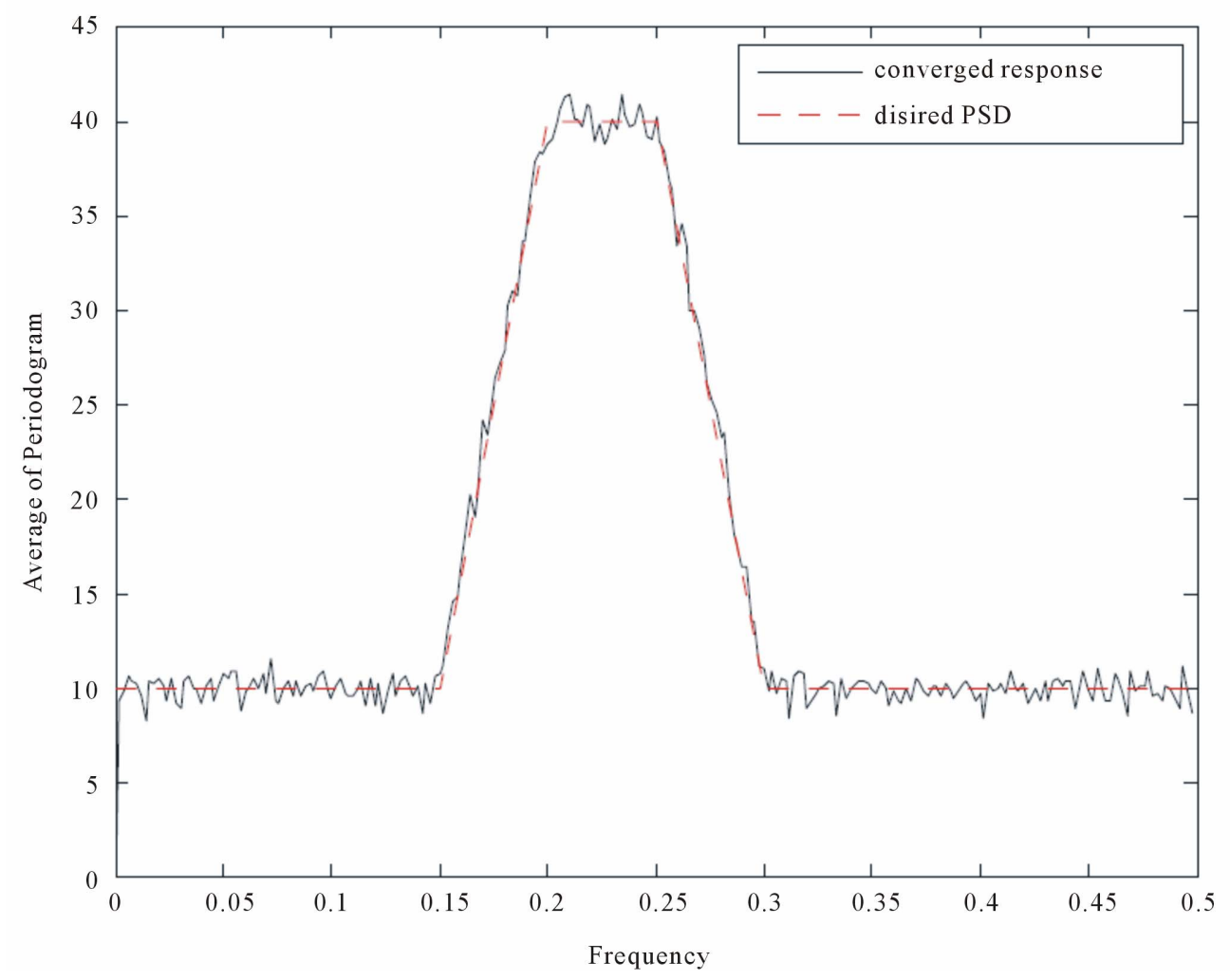

Figure 9. Synthesized power spectral density.

nitely divisible or symmetric. Also with simple computational method we can calculate proper transform function to generate desired PDF from Gaussian PDF with shaped spectrum. Furthermore, this computational method guarantees generated spectrum to be under the wanted spectrum for the first approximation. The error function is reduced to zero by the means of sinusoidal component with proper frequency and amplitude PDF, but with small deviation from exact desired PDF. In other words, there is some tradeoff between exact PDF and PSD. The ergodicity in the mean and variance has been proven under certain conditions. We have avoided bottleneck calculation of Hankel transform function that [8] faces directly to it. Because of negligible deviation from exact PDF it can be used in any practical system.

\section{REFERENCES}

[1] O. Wright and W. Wright, "Flying-Machine," US Patent No. 821393, 1906.

[2] J. K. Wu, "Two Problems of Computer Mechanics Program System," Proceedings of Finite Element Analysis and CAD, Peking University Press, Beijing, 1994, pp. 9-15.

[3] B. Liu and D. Munson, "Generation of a Random Sequence Having a Jointly Specified Marginal Distribution and Autocovariance," IEEE Transaction on Acoustic,
Speech and Signal Processing, Vol. 30, 1982, pp. 973983.

[4] R. Ortega, A. Loria and R. Kelly, “A Semiglobally Stable Output Feedback PI2D Regulator for Robot Manipulators," IEEE Transactions on Automatic Control, Vol. 40, No. 8, 1995, pp. 1432-1436. doi:10.1109/9.402235

[5] E. Wit and J. McClure, "Statistics for Microarrays: Design, Analysis, and Inference," 5th Edition, John Wiley \& Sons Ltd., Chichester, 2004.

[6] A. S. Prasad, "Clinical and Biochemical Spectrum of Zinc Deficiency in Human Subjects," In: A. S. Prasad, Ed., Clinical, Biochemical and Nutritional Aspects of Trace Elements, Alan R. Liss, Inc., New York, 1982, pp. 5-15.

[7] B. M. S. Giambastiani, "Evoluzione Idrologicaed Idrogeologica Della Pineta di San Vitale (Ravenna)," Ph.D. Thesis, Bologna University, Bologna, 2007.

[8] S. Kay, "Representation and Generation of Non-Gaussian Wide-Sense Stationary Random Processes with Arbitrary PSDs and a Class of PDF," IEEE Transaction on Signal Processing, Vol. 58. No. 7, 2010, pp. 3448-3458. doi:10.1109/TSP.2010.2046437

[9] G. L. Wise, A. P. Traganitis and J. B. Thomas, "The Effect of a Memory Less Nonlinearity on the Spectrum of a Random Process," IEEE Transactions on Information Theory, Vol. 23, No. 1, 1977, pp. 84-89.

[10] Bendat and Piersol, "Random Data, Analysis and Measurement Procedures," 3rd Edition, Wiley, New York, 2000 . 


\section{Appendix}

A) Mean value

$$
\begin{aligned}
E & {[Z[n]] } \\
= & E\left[\sum_{i=1}^{M}\left\{A_{i} \cos \left(2 \pi F_{i} n+\varphi_{i}\right)+\frac{L_{n}}{M}\right\}\right] \\
& +j E\left[\sum_{i=1}^{M}\left\{B_{i} \sin \left(2 \pi F_{i}^{\prime} n+\varphi_{i}\right)+\frac{L_{n}^{\prime}}{M}\right\}\right] \\
= & E\left[L_{n}+\sum_{i=1}^{M} A_{i} \cos \left(2 \pi F_{i} n+\varphi_{i}\right)\right] \\
& +j E\left[L_{n}^{\prime}+\sum_{i=1}^{M} B_{i} \cos \left(2 \pi F_{i}^{\prime} n+\varphi_{i}^{\prime}\right)\right] \\
= & E\left[L_{n}\right]+E\left[\sum_{i=1}^{M}\left\{A_{i} \cos \left(2 \pi F_{i} n+\varphi_{i}\right)\right\}\right] \\
& +E\left[L_{n}^{\prime}\right]+j E\left[\sum_{i=1}^{M}\left\{B_{i} \cos \left(2 \pi F_{i}^{\prime} n+\varphi_{i}^{\prime}\right)\right\}\right]
\end{aligned}
$$

$$
\begin{aligned}
& E\left[\sum_{i=1}^{M}\left\{A_{i} \cos \left(2 \pi F_{i} n+\varphi_{i}\right)\right\}\right] \\
& =\sum_{i=1}^{M} E_{A}\left[A_{i}\right] E_{\varphi, F}\left[\cos \left(2 \pi F_{i} n+\varphi_{i}\right)\right]
\end{aligned}
$$

while

$$
\begin{aligned}
& E_{\varphi, F}\left[\cos \left(2 \pi F_{i} n+\varphi_{i}\right)\right] \\
& =E_{F} E_{(\varphi \mid F)}\left[\cos \left(2 \pi F_{i} n+\varphi_{i}\right) \mid F\right] \\
& =E_{F} \int_{-\pi}^{\pi} \cos \left(2 \pi F_{i} n+\varphi\right) \mathrm{d} \varphi=0
\end{aligned}
$$

similarly

$$
E\left[\sum_{i=1}^{M}\left\{B_{i} \cos \left(2 \pi F_{i} n+\varphi_{i}\right)\right\}\right]=0
$$

therefore

$$
E[Z[n]]=E\left[L_{n}\right]+j E\left[L_{n}^{\prime}\right]=E[x]+j E[y]
$$

and

B) Autocorrelation

$$
\begin{aligned}
E\left[Z[n] Z^{*}[n+r]\right]= & E\left[\sum_{i=1}^{M}\left\{A_{i} \cos \left(2 \pi F_{i} n+\varphi_{i}\right)+j B_{i} \cos \left(2 \pi F_{i}^{\prime} n+\varphi_{i}^{\prime}\right)+\frac{L_{n}}{M}+j \frac{L_{n}^{\prime}}{M}\right\}\right. \\
& \left.\times \sum_{i=1}^{M}\left\{A_{i} \cos \left(2 \pi F_{i}(n+r)+\varphi_{i}\right)-j B_{i} \cos \left(2 \pi F_{i}^{\prime}(n+r)+\varphi_{i}^{\prime}\right)+\frac{L_{n+r}}{M}-j \frac{L_{n+r}^{\prime}}{M}\right\}\right] \\
= & E\left[\sum_{k=1}^{M} \sum_{i=1}^{M} A_{i} A_{k} \cos \left(2 \pi F_{i} n+\varphi_{i}\right) \cos \left(2 \pi F_{k}(n+r)+\varphi_{k}\right)\right] \\
& +E\left[\sum_{k=1}^{M} \sum_{i=1}^{M} B_{i} B_{k} \cos \left(2 \pi F_{i}^{\prime} n+\varphi_{i}^{\prime}\right) \cos \left(2 \pi F_{k}^{\prime}(n+r)+\varphi_{k}^{\prime}\right)\right] \\
& +E\left[L_{n} \sum_{i=1}^{M} A_{i} \cos \left(2 \pi F_{i}(n+r)+\varphi_{i}\right)\right]+E\left[L_{n+r} \sum_{i=1}^{M} A_{i} \cos \left(2 \pi F_{i}(n)+\varphi_{i}\right)\right] \\
& +E\left[L_{n} L_{n+r}\right]+E\left[L_{n}^{\prime} L_{n+r}^{\prime}\right]
\end{aligned}
$$

the above relation can be simplified as

$$
\begin{aligned}
& E\left[\sum_{k=1}^{M} \sum_{i=1}^{M} \frac{A_{i} A_{k}}{2}\left\{\cos \left(2 \pi F_{i} n+2 \pi F_{k}(n+r)+\varphi_{k}+\varphi_{i}\right)+\cos \left(2 \pi F_{i} n-2 \pi F_{k}(n+r)+\varphi_{i}-\varphi_{k}\right)\right\}\right] \\
& =\sum_{j=1}^{M} \sum_{i=1}^{M} E\left[\frac{A_{i} A_{k}}{2}\right] \\
& E\left[\cos \left(2 \pi F_{i} n-2 \pi F_{k}(n+r)+\varphi_{i}-\varphi_{j}\right)\right] \delta_{i k}=\sum_{i=1}^{M} E\left[\frac{A_{i} A_{i}}{2}\right] E\left[\cos \left(2 \pi F_{i} r\right)\right] \\
& =\sum_{i=1}^{M} E\left[\frac{A_{i}^{2}}{2}\right]_{0}^{\frac{1}{2}} \cos (2 \pi f r) p_{F}(f) \mathrm{d} f=\frac{M}{2} E\left[A^{2}\right] \int_{0}^{\frac{1}{2}} \cos (2 \pi f r) p_{F}(f) \mathrm{d} f
\end{aligned}
$$

similarly 


$$
\begin{aligned}
& E\left[\sum_{k=1}^{M} \sum_{i=1}^{M} \frac{B_{i} B_{k}}{2}\left\{\cos \left(2 \pi F_{i}^{\prime} n+2 \pi F_{k}^{\prime}(n+r)+\varphi_{k}^{\prime}+\varphi_{i}^{\prime}\right)+\cos \left(2 \pi F_{i}^{\prime} n-2 \pi F_{k}^{\prime}(n+r)+\varphi_{i}^{\prime}-\varphi_{k}^{\prime}\right)\right\}\right] \\
& =\frac{M}{2} E\left[B^{2}\right] \int_{0}^{\frac{1}{2}} \cos \left(2 \pi f^{\prime} r\right) p_{F}\left(f^{\prime}\right) \mathrm{d} f^{\prime}
\end{aligned}
$$

on the other hand

$$
\begin{aligned}
& E\left[L_{n} \sum_{i=1}^{M} A_{i} \cos \left(2 \pi F_{i}(n+r)+\varphi_{i}\right)\right]=0 \\
& E\left[L_{n+r} \sum_{i=1}^{M} A_{i} \cos \left(2 \pi F_{i}(n+r)+\varphi_{i}\right)\right]=0
\end{aligned}
$$

$$
\begin{aligned}
& E_{\varphi}\left[\exp \left(j \omega\left\{A_{i} \cos \left(2 \pi F_{i} n+\varphi_{i}\right)+\frac{L_{n}}{M}\right\}\right)\right] \\
& =\int_{-\pi}^{\pi} \exp \left(j \omega A_{i} \cos \left(2 \pi F_{i} n+\varphi_{i}\right)\right) \frac{1}{2 \pi} \mathrm{d} \varphi=J_{0}\left(\omega A_{i}\right)
\end{aligned}
$$

and

$$
\begin{aligned}
& \mathfrak{B}_{r}=E\left[L_{n} L_{n+r}\right] \\
& \mathfrak{B}_{r}^{\prime}=E\left[L_{n}^{\prime} L_{n+r}^{\prime}\right]
\end{aligned}
$$

therefore

$$
\begin{aligned}
& E[Z[n] Z[n+r]] \\
= & \frac{M}{2} E\left[A^{2}\right] \int_{0}^{\frac{1}{2}} \cos (2 \pi f r) p_{F}(f) \mathrm{d} f \\
+ & \frac{M}{2} E\left[B^{2}\right] \int_{0}^{\frac{1}{2}} \cos \left(2 \pi f^{\prime} r\right) p_{F}\left(f^{\prime}\right) \mathrm{d} f^{\prime}+\mathfrak{B}+\mathfrak{B}^{\prime}
\end{aligned}
$$

C) PDF of $A$ and $B$

Assume $L_{n}, L_{n}^{\prime}$ are constant values, with this assumption

$$
\begin{aligned}
\left.\Psi_{\operatorname{Re}\{Z[n]\}}\right|_{L_{n}} & =E\left[\exp \left(\left.j \omega \operatorname{Re}\{Z[n]\}\right|_{L_{n}}\right)\right] \\
& =E_{A} E_{F} E_{\varphi}\left[\exp \left(j \omega \sum_{i=1}^{M} A_{i} \cos \left(2 \pi F_{i} n+\varphi_{i}\right)\right)\right] \\
\left.\Psi_{\operatorname{Im}\{Z[n]\}}\right|_{L_{n^{\prime}}} & =E\left[\exp \left(\left.j \omega \operatorname{Im}\{Z[n]\}\right|_{L_{n}}\right)\right] \\
& =E_{B} E_{F^{\prime}} E_{\varphi^{\prime}}\left[\exp \left(j \omega \sum_{i=1}^{M} B_{i} \cos \left(2 \pi F_{i}^{\prime} n+\varphi_{i}^{\prime}\right)\right)\right] \\
\left.\Psi_{\operatorname{Re}\{Z[n]\}\}}\right|_{L_{n}} & =\prod_{i=1}^{M} E\left[\exp \left(j \omega A_{i} \cos \left(2 \pi F_{i} n+\varphi_{i}\right)\right)\right] \\
& =E\left[\exp \left(j \omega A_{i} \cos \left(2 \pi F_{i} n+\varphi_{i}\right)\right)\right]^{M}
\end{aligned}
$$

and

$$
E_{A} E_{F}\left[J_{0}\left(\omega A_{i}\right)\right]=\int_{0}^{\infty} p_{A}(a) J_{0}\left(\omega A_{i}\right) \mathrm{d} a
$$

Therefore, $\Psi_{\operatorname{Re}\{Z[n]\}} \mid L_{n}$ in $\Delta x$ domain can be modeled as

$$
\Psi_{\operatorname{Re}\{Z[n]\}} \mid L_{n}=\left[\int_{0}^{\infty} p_{A}(a) J_{0}(\omega a) \mathrm{d} a\right]^{M}
$$

by considering Bessel function characteristics we have

$$
\int_{0}^{\infty} J_{0}(a v) J_{0}\left(a^{\prime} v\right) v \mathrm{~d} v=\frac{\delta\left(a-a^{\prime}\right)}{a}
$$

Therefore

$$
\begin{aligned}
& {\left[\Psi_{\operatorname{Re}\{Z[n]\}} \mid L_{L_{n}}\right]^{\frac{1}{M}}=\int_{0}^{\infty} p_{A}(a) J_{0}(\omega a) \mathrm{d} a} \\
& =\int_{0}^{\infty} \omega J_{0}\left(\omega a^{\prime}\right)\left[\left.\Psi_{\operatorname{Re}\{Z[n]\}}\right|_{L_{n}}\right]^{\frac{1}{M}} \mathrm{~d} \omega \\
& =\int_{0}^{\infty} \omega J_{0}\left(\omega a^{\prime}\right) \int_{0}^{\infty} p_{A}(a) J_{0}(\omega a) \mathrm{d} a \mathrm{~d} \omega \\
& =\int_{0}^{\infty} p_{A}(a) \int_{0}^{\infty} \omega J_{0}(\omega a) J_{0}(\omega a) \mathrm{d} \omega \mathrm{d} a \\
& =\int_{0}^{\infty} p_{A}(a) \frac{\delta\left(a-a^{\prime}\right)}{a} \mathrm{~d} a \\
& =\frac{p_{A}\left(a^{\prime}\right)}{a^{\prime}}
\end{aligned}
$$

if we consider

suddenly

$$
\begin{aligned}
& \Psi(\omega)=E\left[\exp \left(j \omega A_{i} \cos \left(2 \pi F_{i} n+\varphi_{i}\right)\right)\right] \\
& \Psi^{\prime}(\omega)=E\left[\exp \left(j \omega B_{i} \cos \left(2 \pi F_{i}^{\prime} n+\varphi_{i}^{\prime}\right)\right)\right]
\end{aligned}
$$

then

$$
\Psi(\omega)=E_{A} E_{F} E_{\varphi}\left[\exp \left(j \omega A_{i} \cos \left(2 \pi F_{i} n+\varphi_{i}\right)\right)\right]
$$

and

$$
p_{A}(a)=a \int_{0}^{\infty}\left[\Psi_{\operatorname{Re}\{Z[n]\}} \mid L_{n}\right]^{\frac{1}{M}} J_{0}(\omega a) \omega \mathrm{d} \omega
$$

similarly

$$
p_{B}(b)=b \int_{0}^{\infty}\left[\left.\Psi_{\operatorname{Im}\{Z[n]\}}\right|_{L_{n}}\right]^{\frac{1}{M}} J_{0}(\omega b) \omega \mathrm{d} \omega
$$

